\title{
Detection of the Impact of Ice Crystal Accretion in an Aircraft Engine Compression System During Dynamic Operation
}

\author{
Ryan D. May* \\ Vantage Partners, LLC, Brook Park, OH, 44142 \\ Donald L. Simon ${ }^{\dagger}$ and Ten-Huei Guo \\ NASA Glenn Research Center, Cleveland, OH, 44135
}

\begin{abstract}
The accretion of ice in the compression system of commercial gas turbine engines operating in high ice water content conditions is a safety issue being studied by the aviation community. While most of the research focuses on the underlying physics of ice accretion and the meteorological conditions in which accretion can occur, a systems-level perspective on the topic lends itself to potential near-term operational improvements. Here a detection algorithm is developed which has the capability to detect the impact of ice accretion in the Low Pressure Compressor of an aircraft engine during steady flight as well as during changes in altitude. Unfortunately, the algorithm as implemented was not able to distinguish throttle changes from ice accretion and thus more work remains to be done.
\end{abstract}

\begin{tabular}{|c|c|}
\hline & Nomenclature \\
\hline C-MAPSS40k & $=$ the Commercial Modular Aero-Propulsion System Simulation $40,000 \mathrm{lb}_{\mathrm{f}}$ \\
\hline EPR & $=$ Engine Pressure Ratio $\left(P_{50} / P_{2}\right)$ \\
\hline$H$ & $=$ fault influence coefficient matrix \\
\hline HIWC & $=$ High Ice Water Content \\
\hline$K$ & $=$ input influence coefficient matrix \\
\hline LPC & $=$ Low Pressure Compressor \\
\hline $\mathrm{LPC}_{\%}$ & $=$ LPC efficiency $(\%)$ \\
\hline $\mathrm{LPC}_{\text {eff }}$ & $=\mathrm{LPC}$ efficiency health parameter \\
\hline $\mathrm{LPC}_{\text {flow }}$ & $=$ LPC flow capacity health parameter \\
\hline $\mathrm{LPC}_{\mathrm{w}}$ & $=\mathrm{LPC}$ mass flow rate $\left(\mathrm{lb}_{\mathrm{m}} / \mathrm{s}\right)$ \\
\hline $\mathrm{LPC}_{\mathrm{nom}}$ & $=$ nominal LPC characteristic \\
\hline$m$ & $=$ vector of input shifts \\
\hline$N_{c}$ & $=$ Core shaft speed $(\mathrm{rpm})$ \\
\hline$N_{f}$ & $=$ Fan shaft speed $(\mathrm{rpm})$ \\
\hline$P_{x}$ & $=$ Pressure at station $x(\mathrm{psia})$ \\
\hline PLA & $=$ Power Lever Angle $(\mathrm{deg})$ \\
\hline$R$ & $=$ sensor noise covariance matrix \\
\hline$T_{x}$ & $=$ Temperature at station $x$ (degree Rankine) \\
\hline$w$ & $=$ vector of sensor measurement noise \\
\hline$x$ & $=$ vector of health parameter shifts \\
\hline$\hat{x}$ & $=$ estimated vector of health parameter shifts \\
\hline$X_{c 2}$ & $=$ Sensor value $X$ corrected to inlet conditions \\
\hline$y$ & $=$ vector of corrected sensor residuals \\
\hline$\Delta \mathrm{X}$ & $=$ Shift in parameter $\mathrm{X}$ (difference between long and short window average \\
\hline
\end{tabular}

\footnotetext{
* Controls Engineer, 3000 Aerospace Parkway, ryan.d.may@nasa.gov

${ }^{\dagger}$ Research Engineer, Controls and Dynamics Branch, donald.l.simon@ @asa.gov, AIAA Member

* Research Engineer, Controls and Dynamics Branch, ten-huei.guo-1@ nasa.gov, AIAA Member
} 


\section{Introduction}

$\mathrm{O}$ VER the past twenty years, there have been over 100 reported cases of aircraft engine power loss due to the accretion of ice crystal particles in the compression system of commercial turbofan engines. ${ }^{1}$ The majority of the work in this area has focused on understanding the mechanism by which particles in high ice-water content (HIWC) conditions can accrete on compressor stator blades and understanding the environmental conditions in which accretion can occur. While avoidance of HIWC conditions and compressor redesigns are the ideal long-term solutions, a systems level analysis highlights some near-term capabilities.

From a propulsion system perspective, the accreted ice can be treated as a change in the effected compressor's map $^{2}$ as shown in Figure 1. In previous work, low pressure compressor (LPC) maps were generated with various levels of ice blockage using a mean-line compressor design code. ${ }^{3}$ By moving between these maps the impact of ice blockage growth can be simulated.

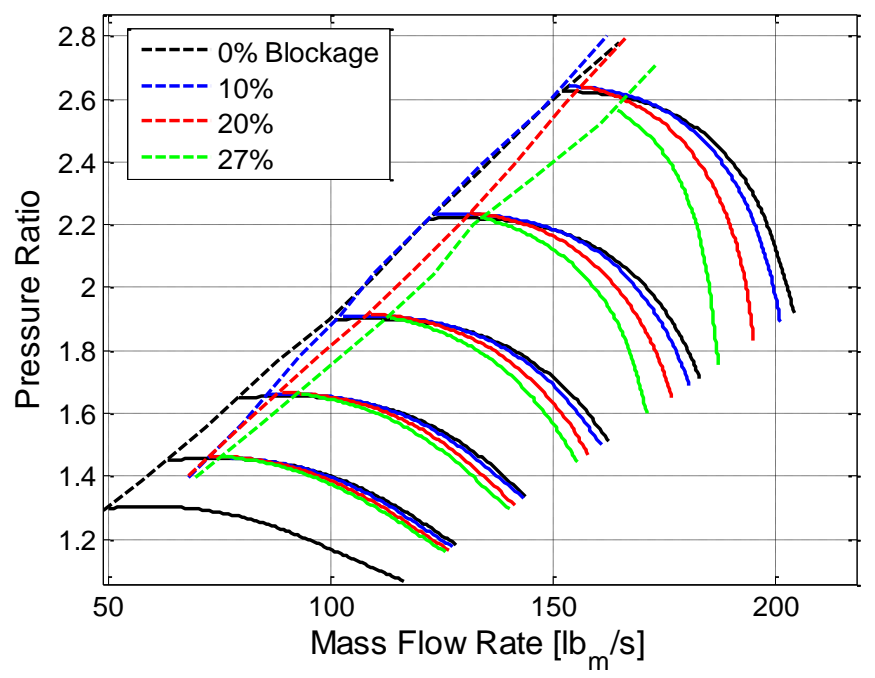

Figure 1. LPC maps used to simulate the impact of ice blockage in the second row stators. Reproduced from Ref. 6 based on data from Ref. 2. Surge lines (dashed) and speed lines (solid) are shown.

Previously, the Commercial Modular Aero-Propulsion System Simulation 40k (C-MAPSS40k), a generic commercial turbofan engine simulation, ${ }^{4}$ was shown to be capable of simulating engine rollback (loss of thrust) when a very simple ice blockage model was used. ${ }^{5}$ The engine simulation's capabilities were upgraded by installing the stacked maps of Figure 1 and allowing the user to input a desired ice blockage level. Desired levels between the discrete levels for which there are maps are found by linear interpolation. Due to the fact that no experimental data exists, this model is unable to be validated at the present time. However, system-level changes should be realistic in direction as well as approximate magnitude. Using this updated simulation, a detection approach was developed that was found to be capable of detecting the effect of ice particle blockage on the LPC performance during steady-state flight (constant altitude, speed, and throttle). ${ }^{6}$ This detection technique uses the two shaft speed sensors as input to a linear estimator in order to estimate the change in LPC performance parameters. The approach is robust enough to handle engines with different levels of deterioration, existing faults, as well as the typical engine-to-engine variation. Importantly, the algorithm is computationally simple enough to allow it to be integrated into a typical engine controller. Unfortunately, approximately half of all icing related power loss events occur during aircraft descent ${ }^{7}$ and thus a detection strategy that does not rely on the steady-state assumption is necessary.

The work in this paper seeks to develop a detection technique that is capable of detecting the change in LPC performance due to ice crystal blockage during both descent and cruise flight conditions. Again, it is critical that the detection algorithm is computationally simple and has low memory requirements so that it can be integrated into modern engine controllers. The approach investigated in this work is an extension of the previous algorithm. Section II describes the new algorithm; while Section III details its application to the C-MAPSS40k engine simulation with embedded LPC maps for modeling ice accretion. Conclusions and future work are discussed in Section IV. 


\section{Detection Algorithm}

Since the accretion of ice in the LPC manifests as a shift in the compressor map, these changes are captured in the LPC health parameters. Health parameters are a common metric used in gas path diagnostic work in order to capture the shift in component performance. For the LPC, the health parameters of interest are efficiency and flow capacity; these parameters $\left(\mathrm{LPC}_{\text {eff }}\right.$ and $\left.\mathrm{LPC}_{\text {flow }}\right)$ are defined as:

$$
\begin{gathered}
\mathrm{LPC}_{\mathrm{w}}=\mathrm{LPC}_{\text {wnom }}\left(1-\mathrm{LPC}_{\text {flow }}\right) \\
\mathrm{LPC}_{\%}=\mathrm{LPC}_{\% \text { nom }}\left(1-\mathrm{LPC}_{\text {eff }}\right)
\end{gathered}
$$

Here, the $w$ subscript refers to mass flow rate, \% is the component efficiency, and nom is the nominal value of the associated parameter. By knowing the current value of the LPC mass flow rate and what the value "should" be for the given operating point, the flow capacity health parameter can be computed.

The approach of the detection algorithm is to estimate the values of the LPC health parameters based on engine sensor data. When these health parameters exceed a specified threshold, an icing event will be declared. One approach to estimating the health parameters given dynamic measurement data is to use a self-tuning, model-based algorithm like the Kalman filter. ${ }^{8}$ Unfortunately, these techniques are both computationally and memory intensive. In order to implement the detection algorithm on the fleet of existing engines, a simpler approach is necessary.

To this end, a linear estimator was previously developed that relied on an assumption that the engine inlet conditions (altitude and Mach number) and throttle are quasi-steady. ${ }^{6}$ As shown in Eq. (3), the vector of corrected sensor residuals $(y)$ are expressed as a linear function of the LPC health parameters $(x)$. The matrix $H$ is called the fault-influence coefficient matrix and $w$ is a vector of sensor measurement noise with zero-mean and a covariance matrix of $R$.

$$
y=H x+w
$$

In a model-based approach, the vector of corrected sensor residuals is usually the difference between the expected value of the sensor and the current (or lightly filtered) measurement. This expected value is based on the current operating condition, the current state of the engine, the engine age, and typical engine-to-engine variation. To simplify this, the vector of sensor residuals is defined here as the difference between a long windowed average of the sensor value and a short windowed average. By using a window that is longer than the period over which ice accretion occurs, a "nominal" value of the sensor is obtained that includes the effects of engine health, operating point, and state without needing to know these explicitly. The purpose of the short window average is to lightly filter the data prior to using it for estimation.

The reason that this approach requires the engine to be in steady-state is that any change in the sensor residuals are assumed to be due to a change in the LPC health parameters. To relax this assumption, we can modify the expression for $y$ to include these engine inputs:

$$
y=H x+K m+w
$$

The new term is the product of the vector of known engine input shifts, $m$, and the input-influence coefficient matrix, $K$. The premise of this modification is to note that changes in the sensor residuals can be due to changes in the engine operating point. Thus, if there is a small change in the engine operating conditions, there will be a linearly related change in the sensor residuals. When there is no change in the sensor residuals, that implies that either both $x$ and $m$ are zero, or $H x$ and $\mathrm{Km}$ are equal and opposite in magnitude. To enable this, $m$ is then defined as the change in inputs from nominal. Therefore $m$ will be computed in the same manner as $y$ : the difference between long window time-averaged inputs and short window time-averaged inputs.

From Eq. (4), the shift in the LPC health parameters can be estimated. Here a simple weighted least squares estimation technique ${ }^{9}$ can be used to arrive at:

$$
\hat{x}=\left(H^{T} R^{-1} H\right)^{-1} *\left(H^{T} R^{-1}(y-K m)\right)
$$

To compute this estimate, values of $R, H$, and $K$ must be known. The sensor noise covariance matrix can be computed analytically if the noise is easily quantifiable or numerically based on historical engine data. Because the sensor noise changes based on operating condition, $R$ will change as the operating point changes. The values of $H$ 
and $K$ will also change based on operating condition due to engine nonlinearities. For a gas turbine engine, the vector of corrected sensor residuals is more accurately described by the nonlinear relationship:

$$
y=f(x, m)+w
$$

The two coefficient matrices are the linearized derivatives of $f$ with respect to their associated arguments. These matrices can be computed numerically using high-fidelity, non-linear engine simulations or actual engine data if available.

The complete procedure for the icing detection algorithm is shown in Figure 2. This process repeats every controller time step. Once the filtered sensor values and inputs are computed, the sensor residuals and input changes are computed. Based on the current operating point and engine state, the two influence coefficient matrices can be computed. With this information, an estimate of the LPC health parameters can be computed. Detection logic is then applied to the estimated health parameters to arrive at a "true" or "false" value. The number of consecutive "true" results is counted, and when they exceed a predefined persistence threshold then an icing event is declared. This threshold is found by running a Monte Carlo study and varying the threshold until the necessary false-positive rate is obtained.

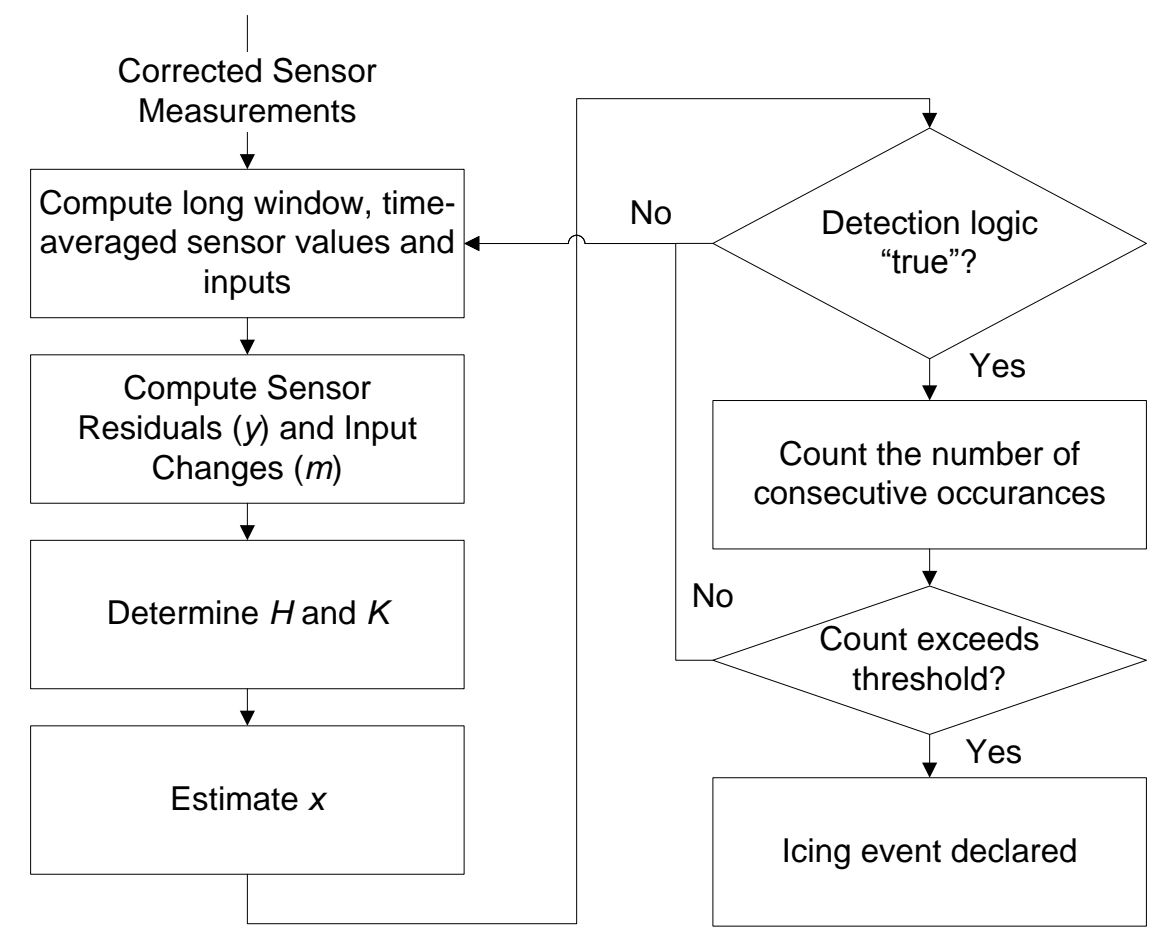

Figure 2. Flow of the ice accretion detection algorithm used in this paper. This process is conducted every controller time step.

In the previous research effort, ${ }^{6}$ two detection logics were applied:

$$
\begin{gathered}
\mathrm{LPC}_{\text {flow }}<\text { thresh }_{1} \\
\mathrm{LPC}_{\text {flow }}^{2}+\mathrm{LPC}_{\text {eff }}^{2}>\text { thresh }_{2}
\end{gathered}
$$

However, the results indicated that the metric of Eq. (7) was significantly better at detecting the impact of ice accretion and detecting events earlier than the sum of squares approach shown in Eq. (8). Thus only Eq. (7) is used in this work. 


\section{Example Application}

For the purpose of developing and testing this detection algorithm, the Commercial Modular Aero-Propulsion System Simulation (C-MAPSS40k v2.3.5) software package is used. C-MAPSS40k is a high-fidelity, publiclyavailable simulation of a generic 40,000lbf thrust class high-bypass turbofan engine (schematic shown in Figure 3). One key feature is that it has a realistic engine controller, thus the control system will respond to changes in engine operation in a reasonable manner. Further, C-MAPSS40k has modular components that are easily modified, making it easy to change the default LPC to use the compressor maps shown in Figure 1 to simulate the effect of ice blockage.

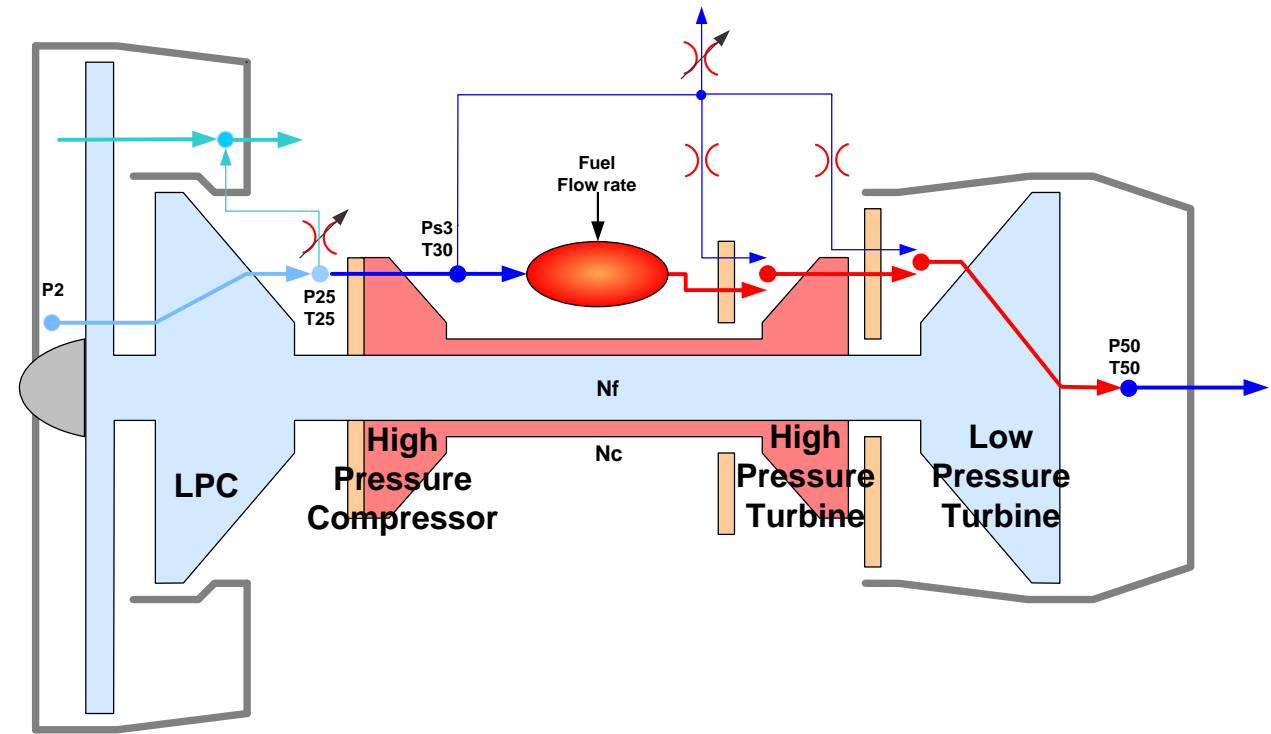

Figure 3. Schematic of the typical twin-spool, high-bypass commercial aircraft engine modeled in C-MAPSS40k. Reproduced from Ref. 3.

The first decision to make when applying this algorithm is to choose the sensors and inputs that will be used. For selecting the sensors, the two primary considerations are: 1) the controlled engine variable and 2) the available sensors. Because the C-MAPSS40k engine is controlled to operate at constant engine pressure ratio (EPR, $\left.P_{50} / P_{2}\right)$, the two shaft speeds will change based on operating condition. In particular, when the LPC performance changes (as it does during ice accretion) this will manifest as a discrepancy between the fan and core shaft speeds. If the engine is fan speed controlled or core speed controlled (the other common configurations), then it does not make sense to use these values in the detection algorithm. In those cases, EPR may be a suitable replacement.

The second consideration is sensor availability. Due to cost and weight constraints, the number of sensors included in the engine is kept to a minimum. To determine which sensors are most sensitive to changes in LPC performance due to ice blockage, a simulation is conducted at various levels of ice blockage and the magnitude of the change in the sensor output is recorded as shown in Figure 4. While this plot is only for one flight condition, many others were simulated and the resulting order of sensor sensitivity is relatively consistent. Unsurprisingly, the most impact is seen in the $P_{25}$ sensor located at the LPC exit. More interesting is the fact that the exhaust gas temperature $\left(T_{50}\right)$ is the second most sensitive of these sensors. 


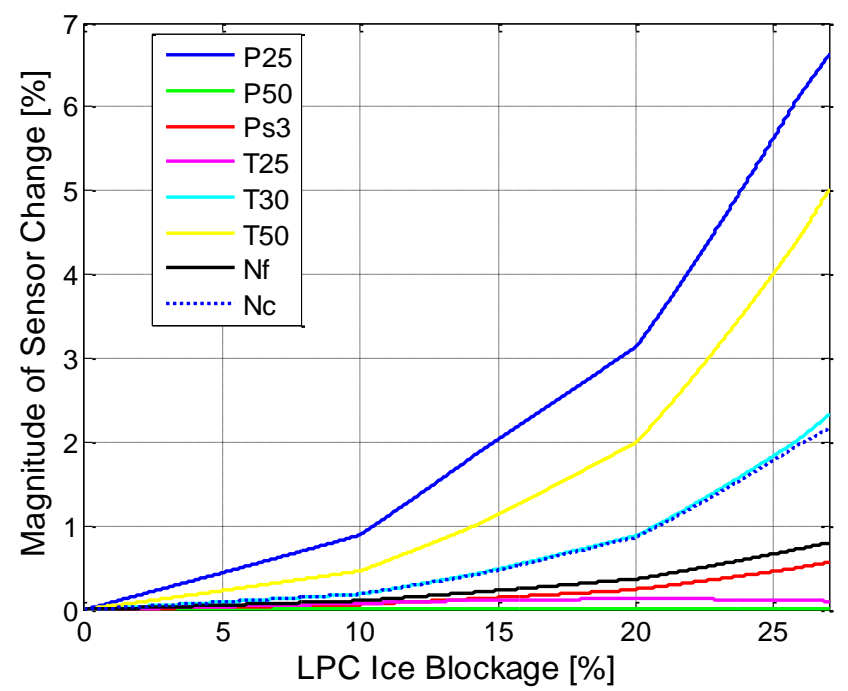

Figure 4. Magnitude of the change in sensor output for all sensors in the C-MAPSS40k engine as the size of the ice blockage in the LPC increases. This data is collected at 10,000ft, Mach 0.6, and a PLA of 68 degrees.

In an effort to make the results from this analysis as generic as possible, the sensors selected to make up the $y$ vector are corrected fan shaft speed $\left(N_{f c 2}\right)$, corrected core shaft speed $\left(N_{c c 2}\right)$, and corrected exhaust gas temperature $\left(T_{50 c_{2}}\right)$. These sensors are available in every high-bypass turbofan engine (unlike the $P_{25}$ sensor, which is less common). In adapting this algorithm to a specific engine, the designer should take advantage of any sensor that produces insight into the impact of the ice blockage.

To choose the inputs comprising the $m$ vector, the designer needs to select inputs such that $\frac{\partial f(x, m)}{\partial m}$ is as linear as possible and such that $m$ is not a function of $x$. Stated another way, it is important that the input vector, $m$, not change as the size of the ice blockage changes. If it does, then that will reduce the detection capability of the algorithm as shifts in the sensor values will not be "assigned" to $x$. For the purposes of this work, the pilot's throttle command (power lever angle, PLA) is selected as the sole element of $m$. It was anticipated that it would be important to also use inlet pressure $\left(P_{2}\right)$, however as the sensor residuals used in the calculations are corrected to sea-level static, standard day conditions, this turns out to not be necessary. Changes in altitude, speed, ambient temperature will lead to very small changes in the elements of $y$ which will translate into very small changes in the LPC health parameters as will be shown later in this paper.

With these initial design decisions completed, work turns to developing the $H$ and $K$ matrices. To determine the value of the elements of $H$ (here a $3 \times 2$ matrix), the nonlinear engine simulation is utilized. A series of tests is conducted at operating points throughout the expected icing envelope $(10,000 \mathrm{ft}$ through $30,000 \mathrm{ft})$ and at each point the two health parameters are individually perturbed and the resulting sensor changes recorded. For example, the $(1,1)$ element of $H$ is the linear impact of a change in LPC efficiency on the corrected fan speed residual, computed as the change in corrected fan speed divided by the size of the perturbation in LPC efficiency. The resulting data is then plotted in Figure 5 as a function of the controlled variable, EPR. The data is then fit with the piece-wise linear curve shown in black. 

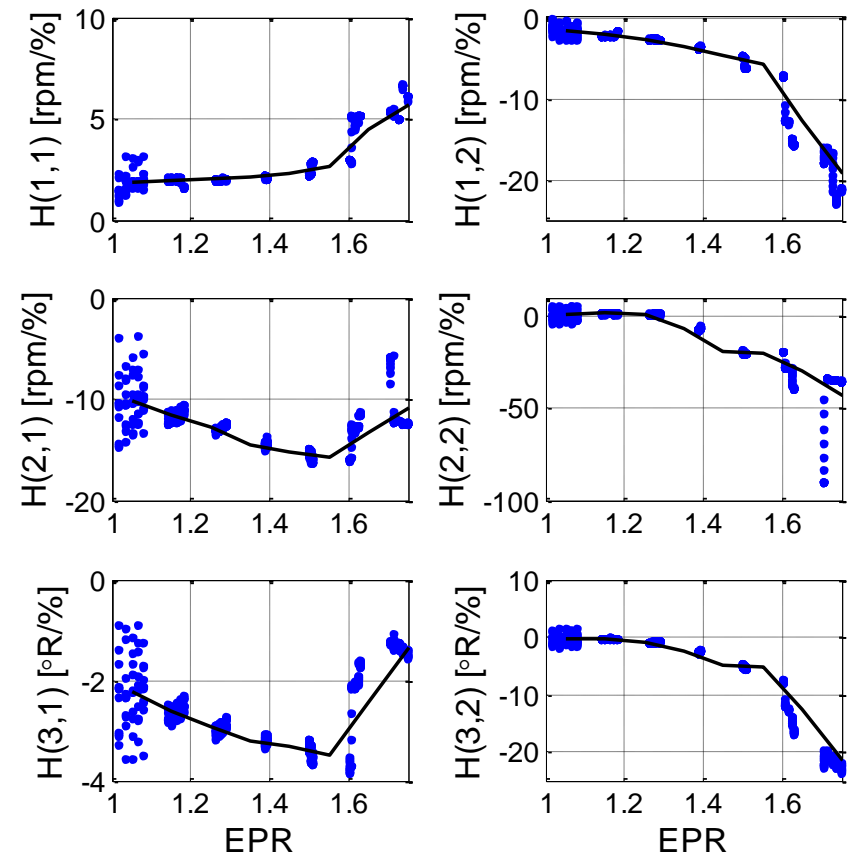

Figure 5. The values of the fault influence coefficient matrix, $H$, for the $C$ MAPSS40k application at various altitude, Mach number, and engine power settings as a function of the engine pressure ratio. The black line represents the piecewise-linear curve fit to the data.

The same process is repeated to determine the input influence coefficient matrix, $K$, as shown in Figure 6. Here, a throttle perturbation of one degree is used to generate the influence matrix. A piece-wise curve is then fit to the data. As with the previous results, the fit is good at mid-range EPR values, however it is particularly poor at high and low EPR values. These piece-wise curves (each comprised of eight points) are then stored in a look-up-table based on EPR. This allows the influence coefficient matrices to be determined quickly with a small memory footprint. 

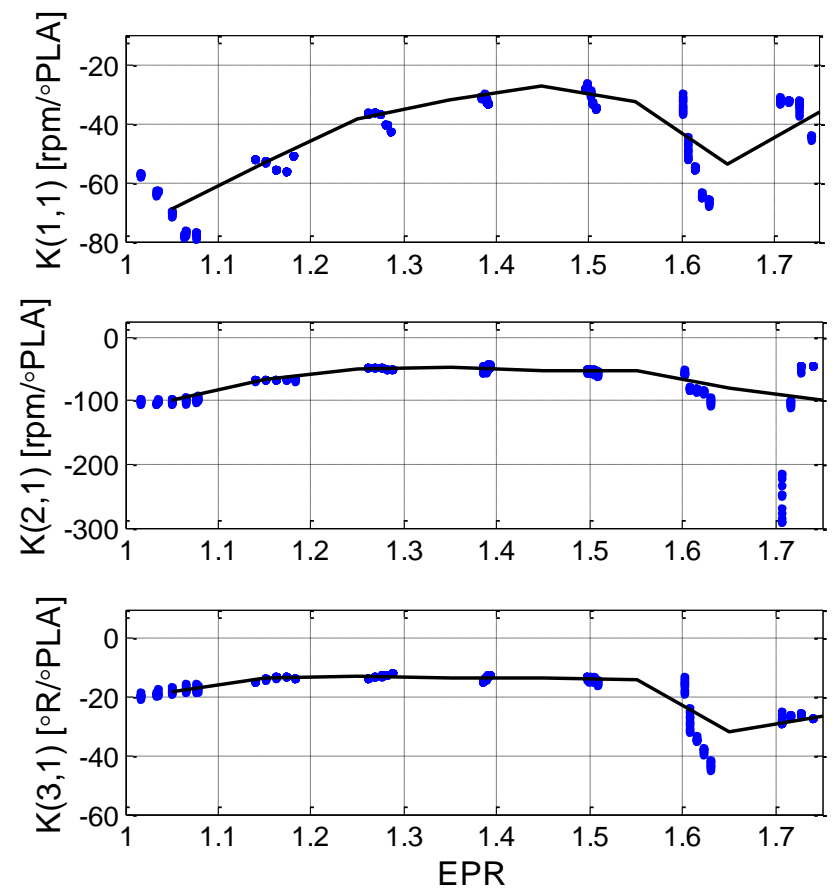

Figure 6. The values of the input influence coefficient matrix, $K$, for the $C$ MAPSS40k application at various altitude, Mach number, and engine power settings as a function of the engine pressure ratio. The black line represents the piecewise-linear curve fit to the data.

In order to test the validity of this approach, three test cases are studied: steady flight, decreasing altitude, and a throttle change. All of these test cases start at a low cruise condition: 10,000ft altitude, Mach 0.6, and a throttle of 68 degrees (nominal EPR of 1.456) and do not include sensor noise. This case was chosen as it is representative of a point that would be traveled through during descent. For each of the test conditions, one test will have no ice accretion, the other will have a five minute accretion from $0 \%$ blockage to $27 \%$ blockage of the LPC. Figure 7 shows the estimation algorithm's results for the case of steady flight. When no ice accretion is present there is no shift in the sensor outputs or in the inputs, thus the estimated health parameter shifts are zero. When ice accretion occurs, there is a shift in the sensor residuals and because there is no change in the inputs, all of the change in residuals is associated with a shift in the health parameters. 

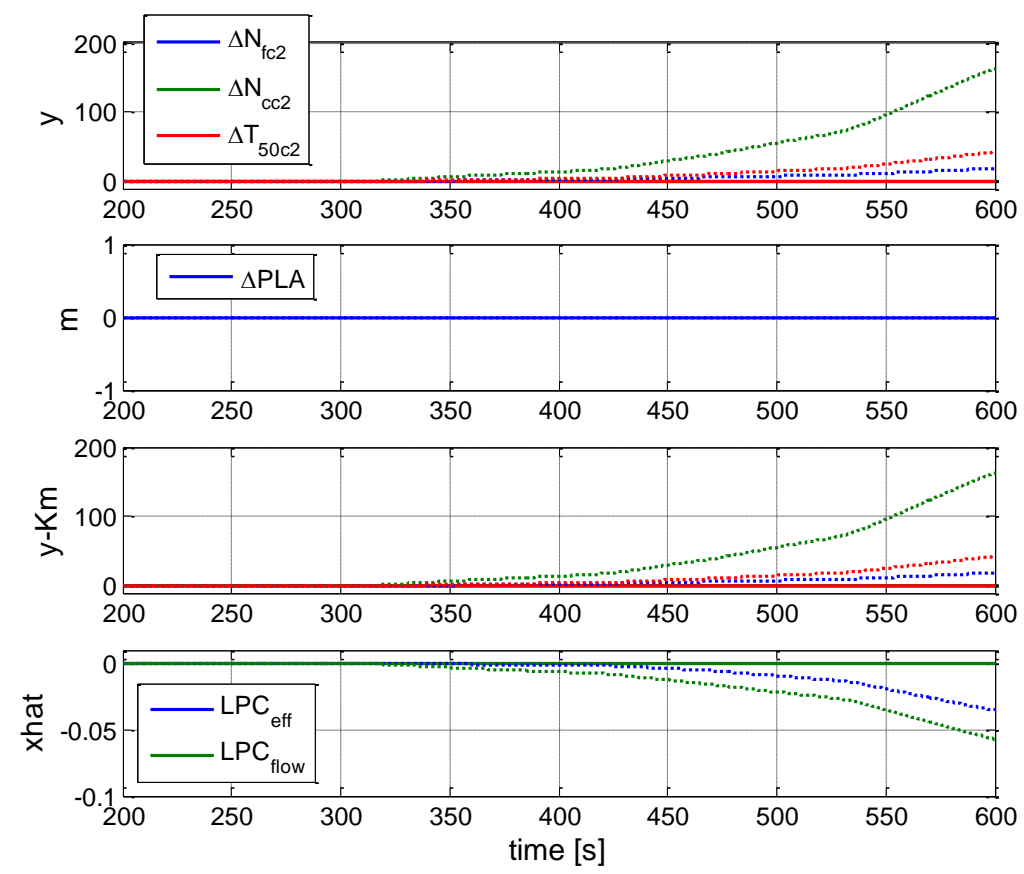

Figure 7. Estimation algorithm output during steady-state test condition with no ice accretion (solid lines) and ice accretion starting at $\mathbf{3 0 0}$ seconds (dotted lines).

The second test case is steady-state operation until 300 seconds at which point the altitude decreases from 10,000ft to 8,000ft over five minutes. The estimator outputs are shown in Figure 8. Due to the fact that the sensor residuals are corrected to sea level conditions, there is only a small shift in the $y$ vector during the non-iced test case which can be seen in Figure 9. Thus, the results are nearly identical to the steady-state test case.
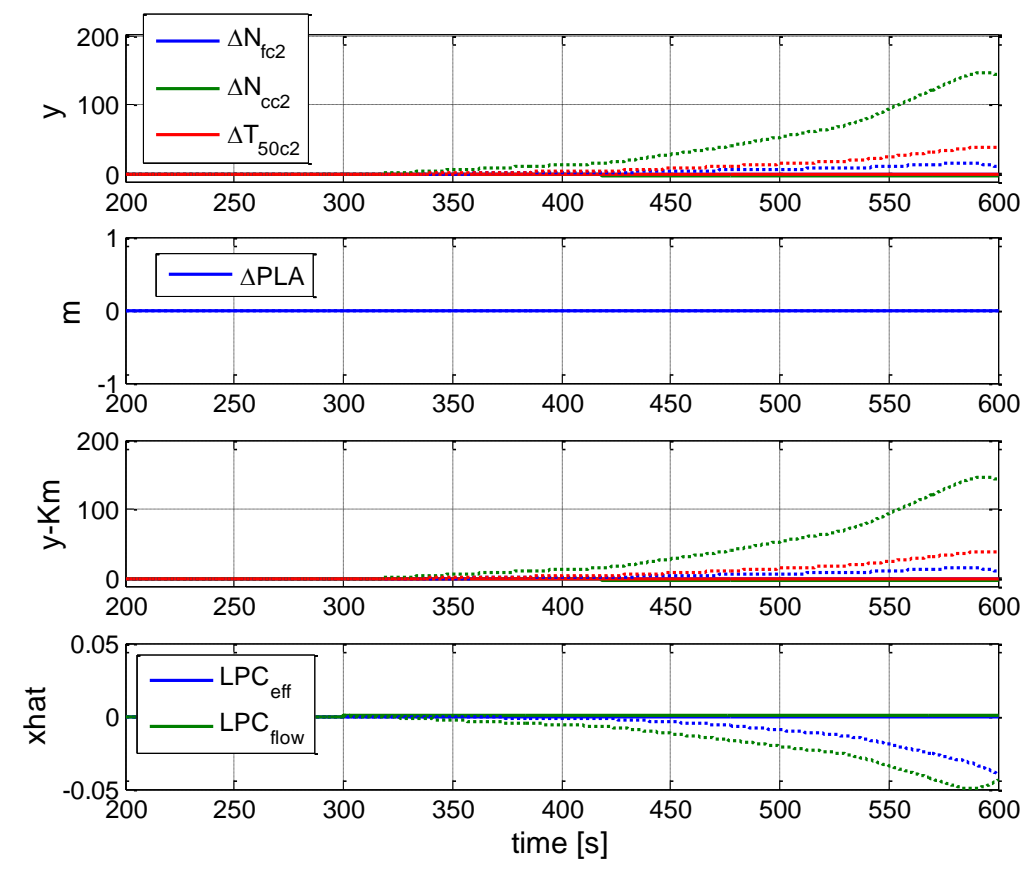

Figure 8. Estimation algorithm outputs during a decrease in altitude starting at 300 seconds. Cases with no ice accretion (solid lines) and ice accretion starting at 300 seconds (dotted lines) are shown. 

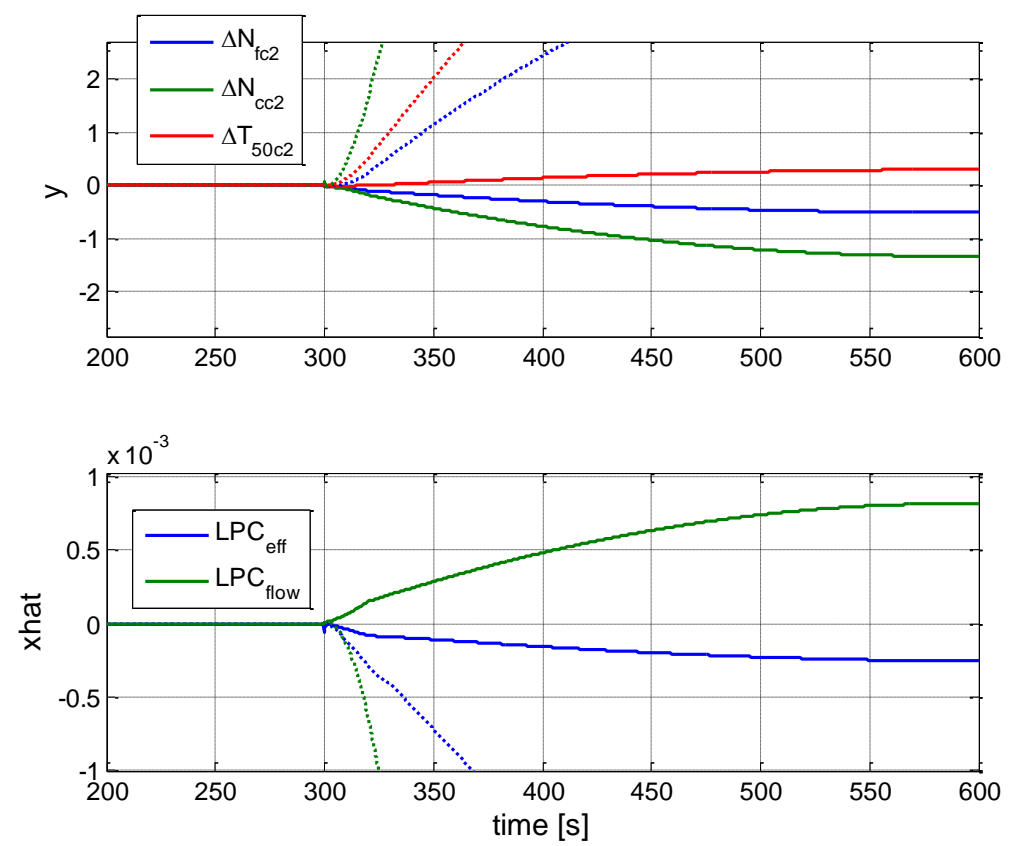

Figure 9. A tighter view of the input and output of the estimation algorithm during a decrease in altitude starting at 300 seconds. Cases with no ice accretion (solid lines) and ice accretion starting at 300 seconds (dotted lines) are shown.

The final test case is at level flight, but at 300 seconds the throttle is ramped down from 68 degrees to 60 degrees over 100 seconds. As shown in Figure 10, the throttle change causes a large shift in corrected sensor residuals. Once the impact of the throttle change is removed, the remainder of the sensor residuals $(y-K m)$ is an order of magnitude smaller. If the influence matrix was exactly correct, the resulting values would be zero for the non-iced case. However, the approximation necessary to determine $K$ is not exact, thus there is a change in the estimates of the LPC health parameters, even for the non-iced case. Here the flow capacity is estimated to increase and the efficiency decreases during the throttle change and as the ice blockage becomes larger, its effect can be seen more clearly. Eventually the erroneous health parameters return to zero while those computed during ice accretion continue to diverge. 

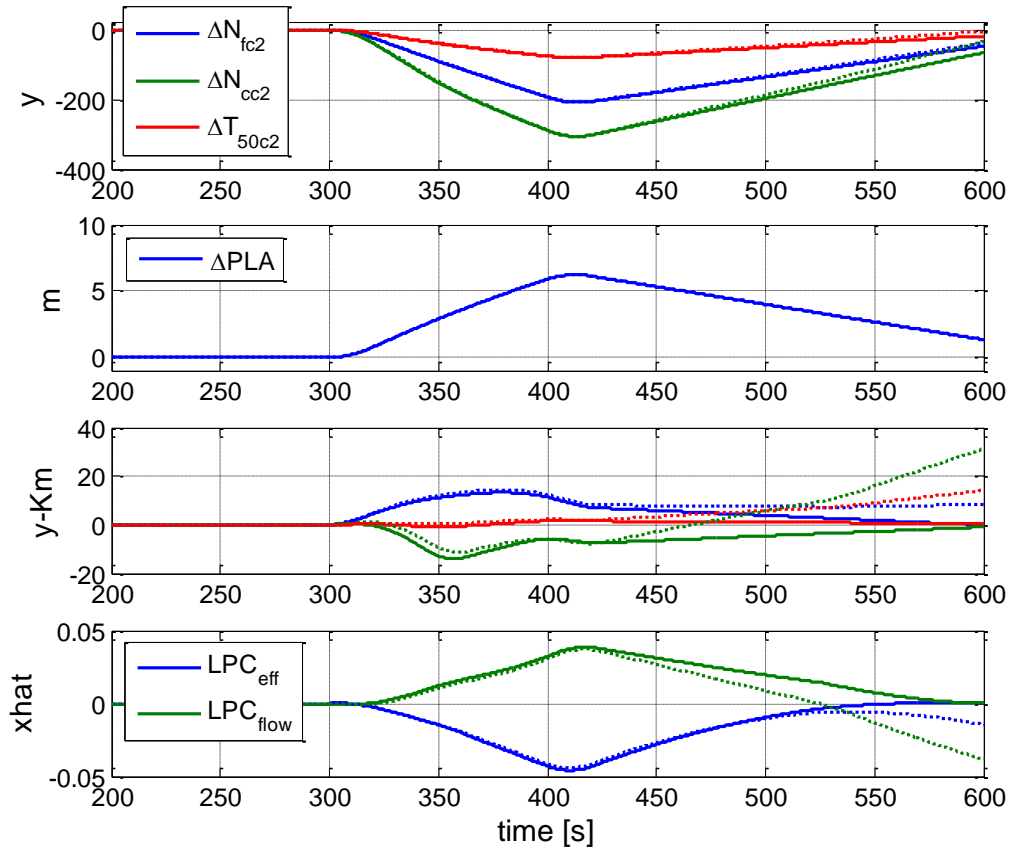

Figure 10. Estimation algorithm outputs during a decrease in throttle starting at 300 seconds until 400 seconds. Cases with no ice accretion (solid lines) and ice accretion starting at $\mathbf{3 0 0}$ seconds (dotted lines) are shown.

Based on these results, it is anticipated that some flight conditions may result in a decrease in the estimated $\mathrm{LPC}_{\text {flow }}$ during non-iced conditions. This presents a problem as this decrease in flow capacity is the detection metric we are using to trigger the declaration of an icing event as shown in Eq. (7). Thus in order to prevent false-positives, the threshold must be set to a more negative value. To determine if this does occur, it is necessary to run the estimation against "realistic" data to determine the appropriate threshold value. To this end, sample flight data (altitude, Mach number, throttle, ambient temperature) from 74 regional jet flights ${ }^{\S}$ was used as inputs to C-MAPSS40k. The software would then simulate the performance of the engines in these conditions (including realistic simulated sensor noise) ${ }^{8}$ The resulting sensor data is run through the detection algorithm using different threshold values in order to determine the detection threshold that produces zero false-positives. The minimum estimated value of the $\mathrm{LPC}_{\text {flow }}$ health parameter during cruise and descent is shown in Figure 11. For the 74 cases analyzed, the minimum estimated value of $\mathrm{LPC}_{\text {flow }}$ is -1.00 . The desired false positive rate for a system like this is very low (less than $0.01 \%$ of flights). Because we do not have enough data, the best we can do is to estimate the maximum false positive rate to be $1 / 75 \approx 1.3 \%$ when the detection threshold is set to be less than -1.00 . With the threshold set this low, none of the previously shown test cases would have resulted in detection. The result of previous work ${ }^{6}$ indicated that a detection threshold of -0.006 was required to achieve a $100 \%$ true-positive detection rate. Thus, the implementation presented here will not adequately detect ice accretion in the low pressure compressor due to inaccuracies in the input influence coefficient matrix, $K$.

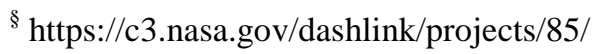




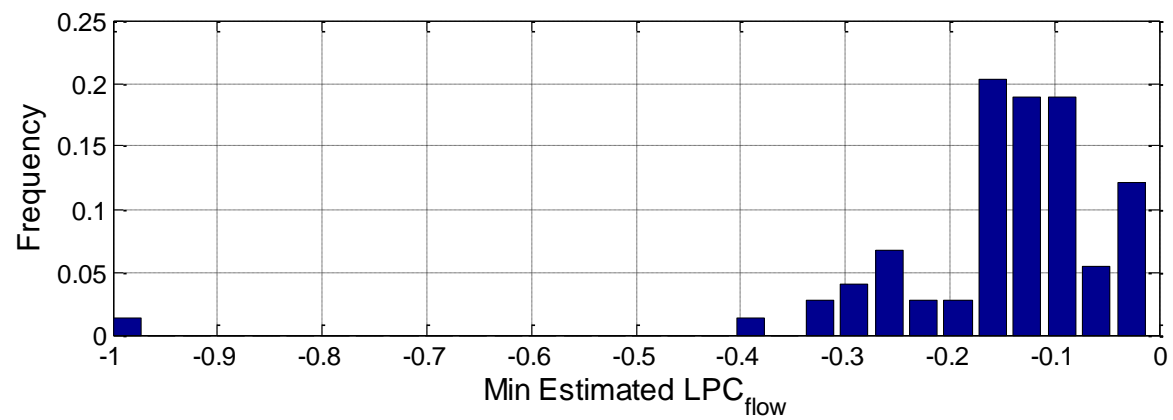

Figure 11. Histogram of the minimum estimated LPCflow health parameter during cruise and descent for 74 sample data flights.

\section{Conclusion}

A simple, linear detection algorithm has been developed with the goal of detecting the impact of ice accreting in the low pressure compressor of a high-bypass turbofan engine during operational transients. The algorithm was applied to the C-MAPSS40k engine simulation and three proof-of-concept tests were conducted showing that for a single operating point it is possible to distinguish the impact of ice accretion from the change in engine performance due to changes in altitude and throttle. Unfortunately, when a study of real flight data was conducted, it was found that the approximate models used in the detection algorithm are not sufficiently precise to achieve the required falsepositive rate.

The approach is promising, however a more precise methodology to compute the input influence coefficient matrix, $K$, is required. Kalman filter-based approaches would likely be successful at the cost of increased processor and memory utilization. Also, it is possible that increasing the dimensionality of $K$ beyond solely EPR, would prove useful. These issues should be considered in any related future work. Additionally, work is on-going to migrate this detection algorithm to an aircraft engine for which experimental data does exist.

\section{Acknowledgments}

The authors would like to thank the NASA Aviation Safety Program's Vehicle Systems Safety Technologies Project for funding this work.

\section{References}

${ }^{1}$ Mason, J., "Current Perspectives on Jet Engine Power Loss in Ice Crystal Conditions: Engine Icing," Presentation at 2008 AIAA Atmospheric and Space Environments, June 23rd, 2009.

${ }^{2}$ Jorgenson, P.C.E., Veres, J.P., May, R.D., Wright, W.B., "Engine Icing Modeling and Simulation (Part I): Ice Crystal Accretion on Compression System Components and Modeling its Effects on Engine Performance," 2011-380025, SAE International Conference on Aircraft and Engine Icing and Ground Deicing, Chicago, IL, Jun 13-17, 2011. doi:10.4271/2011-38-0025

${ }^{3}$ Veres, J.P., "Axial and Centrifugal Compressor Mean Line Flow Analysis," AIAA 2009-1641, $47^{\text {th }}$ AIAA Aerospace Sciences Meeting, Orlando, FL, January, 2009.

${ }^{4}$ May, R.D., Csank, J., Lavelle, T.M., Litt, J.S., and Guo, T-H., “A High-Fidelity Simulation of a Generic Commercial Aircraft Engine and Controller," AIAA-2010-6630, 2010 AIAA Joint Propulsion Conference, Nashville, TN, July, 2010.

${ }^{5}$ May, R.D., Guo, T-H., Veres J.P., Jorgenson, P.C.E., "Engine Icing Modeling and Simulation (Part 2): Performance Simulation of Engine Rollback Phenomena," 2011-38-0026, SAE International Conference on Aircraft and Engine Icing and Ground Deicing, Chicago, IL, Jun 13-17, 2011. doi:10.4271/2011-38-0026

${ }^{6}$ May, R.D., Guo, T-H., Simon, D.L., "An Approach to Detect and Mitigate Ice Particle Accretion in Aircraft Engine Compression Systems," ASME-GT2013-95049, ASME TurboExpo 2013, San Antonio, TX, June 3-7, 2013.

${ }^{7}$ Mason, J.G., Strapp, J.W., Chow, P., “The Ice Particle Threat to Engines in Flight,” AIAA 2006-206, 44 ${ }^{\text {th }}$ AIAA Aerospace Sciences Meeting, Reno, NV, Jan 9-12, 2006.

${ }^{8}$ Armstrong, J.B., Simon, D.L., "Implementation of an Integrated On-Board Aircraft Engine Diagnostic Architecture," AIAA-2011-5859, NASA/TM-2012-217279, 47th AIAA Joint Propulsion Conference and Exhibit, San Diego, California, July 31-August 3, 2011.

${ }^{9}$ Kay, S., Fundamentals of Statistical Signal Processing, Volume 1: Estimation Theory, Prentice Hall, 1993. 